\title{
Effect of patient bladder voiding on radiation dose rates measured around patients undergoing PET/CT imaging using ${ }^{18}$ F-FDG
}

\section{Khaled Soliman and Ahmed Alenezi}

Department of Medical Physics, Prince Sultan Military Medical City, Riyadh, Saudi Arabia

*Corresponding authors: Ahmed Alenezi, Senior Consultant, Department of Medical Physics, Prince Sultan Military Medical City, P.O.Box 7897 (W-913), Riyadh-11159, Saudi Arabia, Tel: +00966114777714 ext 25268; E-mail: alenezi_ahmed@yahoo.co.uk

Received date: Oct 10, 2015, Accepted date: Dec 29, 2015, Publication date: Jan 04, 2016

Copyright: ( 2016 Soliman K, et al. This is an open-access article distributed under the terms of the Creative Commons Attribution License; which permits unrestricted use; distribution; and reproduction in any medium; provided the original author and source are credited.

\begin{abstract}
Accurate dose rate estimates is important for radiation protection specialists conducting risk assessments and performing dose reconstruction in cases of accidental exposures.

Objectives: The objectives of this work was to experimentally evaluate the bladder voiding factor effect on the dose rate measured from patients undergoing PET/CT imaging studies using ${ }^{18} \mathrm{~F}-\mathrm{FDG}$ by directly measuring the dose rate immediately before and after voiding, and compare the results with the current scientific literature.

Results: The bladder voiding effect had a dose rate reduction factor of about $12 \%$ between dose rates measured before and after voiding. This measured reduction factor agreed with the $15 \%$ reported by the AAPM Task Group 108. We have also measured dose rates at one meter from 50 patients and found an average dose rate per unit activity of $93.7 \mu \mathrm{Sv} / \mathrm{hr} / \mathrm{GBq}$. Our dose rate results were in excellent agreement with the results of current published data (92 $\mu \mathrm{Sv} / \mathrm{hr} / \mathrm{GBq}$, AAPM Task Group 108).
\end{abstract}

Conclusions: The presented data can be applied in radiation protection optimization procedures, especially for the protection of the care givers from patients undergoing 18F-FDG PET/CT imaging when they are considered as external radiation source or hazard to others.

The provided information will benefit medical physicist working in nuclear medicine and radiation safety policy makers.

Keywords: ${ }^{18}$ F-FDG; Measured dose rate; Patient voiding factor; $\mathrm{PET} / \mathrm{CT}$

\section{Introduction}

The estimated dose rate at certain distance from a radioactive source depends on the dose rate constant, the source activity and the distance between the source and the measurement point. Once the radioactivity is incorporated into the patient it will additionally depend on patient's body tissues attenuation properties. ${ }^{18} \mathrm{~F}-\mathrm{FDG}$ is a nonspecific tracer mainly used for metabolic activity and concentrates in metabolically active tumors and accumulates in areas with high metabolism such as brain, heart and active muscles. When ${ }^{18} \mathrm{~F}-\mathrm{FDG}$ is injected in patients the non-metabolized ${ }^{18} \mathrm{~F}-\mathrm{FDG}$ is eliminated from the body by glomerular filtration without being resorbed by the renal proximal tubules. Then the eliminated ${ }^{18} \mathrm{~F}-\mathrm{FDG}$ remained and accumulated in the bladder.

Voiding the bladder is recommended to patients by nuclear medicine staff and clinical protocols since it will reduce the patients' bladder dose by eliminating the unused activity of ${ }^{18} \mathrm{~F}-\mathrm{FDG}$ by the patient body; avoid signal interference due to gamma emissions from the bladder and reduce the exposure rate measured from the patient by a certain percentage. Therefore patients are asked to void at the end of the uptake time and before scanning.
In most cases the patient will void prior to imaging, removing approximately 15\%-20\% [1] of the administered activity and thereby decreasing the dose rate by a factor of 0.85 [1].

The objectives of this work was to quantify the effect of patients' bladder emptying on the measured dose rate value by directly measuring the dose rate from the patients immediately before and after voiding, then calculating the ratio of after to before in order to represent the reduction factor in percentage form as described below in the text.

\section{Materials and Methods}

The measured dose rate at voiding time divided by the activity calculated at the voiding time and corrected for radioactive decay was used for each patient to calculate the dose rate per unit activity constant.

Radiation dose measurements were done immediately before and after voiding, in order to calculate the dose rate reduction factor using equation 3 below. We have excluded from this analysis the patients with measured dose rate after voiding that were slightly higher than before voiding due to some mild urine contamination on their clothes. The total number of patients measurements reported in this study is 50, details are in Table 1. 
Citation: Soliman K, Alenezi A (2016) Effect of patient bladder voiding on radiation dose rates measured around patients undergoing PET/CT imaging using ${ }^{18}$ F-FDG. J Nucl Med Radiat Ther 7: 272. doi:10.4172/2155-9619.1000272

Page 2 of 4

\begin{tabular}{|c|c|c|c|c|c|}
\hline Parameter & Average & Standard deviation & $\begin{array}{l}\text { Coefficient of variation } \\
\text { (\%) }\end{array}$ & Minimum & Maximum \\
\hline Weight in [kg] & 76 & 27 & 36 & 15 & 171 \\
\hline Body Mass Index (BMI) & 29 & 9 & 31 & 11 & 58 \\
\hline Injected activity in [MBq] & 326 & 86 & 26 & 147 & 485 \\
\hline Time before voiding in [min] & 39 & 8 & 21 & 17 & 68 \\
\hline Dose reduction due to voiding in [\%] & 12 & 6 & 50 & 2 & 30 \\
\hline $\begin{array}{l}\text { Measured Dose rate per unit activity } \\
\text { in }[\mu \mathrm{Sv} / \mathrm{hr} / \mathrm{GBq}]\end{array}$ & 93.7 & 14 & 15 & 65 & 136 \\
\hline
\end{tabular}

Table 1: Patient data, we have 50 patients in this study. Coefficient of variation is the ratio of the standard deviation over the average.

The radiation dose rate was measured using a calibrated ionisation chamber (SmartIon Type: 2120G; thermo Franklin, Massachusetts, USA). The ${ }^{18} \mathrm{~F}$-FDG dose was administered using an automatic dose injector (Intego, by MedRad Inc, Indianola, PA, USA).

The measurements were taken at one meter from the patient body surface to the entrance of the ionization chamber type radiation detector by marking the floor to show the exact standing positions to be able to reproduce the measurement geometry with ease and accuracy. The patient and the nuclear medicine technologist performing the measurements are in standing position and the radiation detector was aimed at waist level of the patient as illustrated in Figure 1.

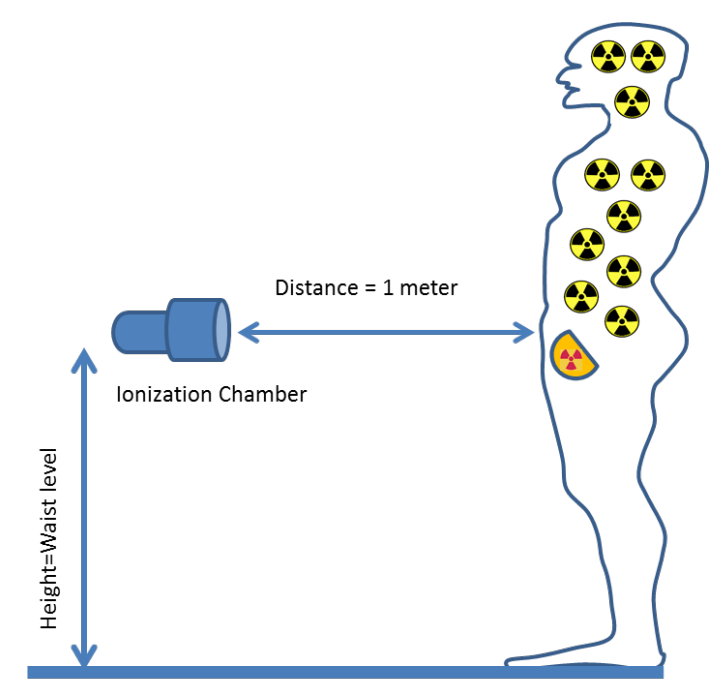

Figure 1: Setup of measurements.

\section{Uptake time correction factor}

The activity measured at the uptake time was calculated using the following relation:

$$
A\left(t_{\text {up }}\right)=A_{0} e^{-\lambda t} u p(1)
$$

Where $\left(t_{\text {up }}\right)$ is the uptake time in minutes, and $(\lambda)$ is equal to $\ln$ $(2) / T_{1 / 2}$.

$\mathrm{T}^{1 / 2}$ is the half-life of ${ }^{18} \mathrm{~F}=110$ minutes.

\section{Dose rate reduction due to voiding}

We have calculated the patient voiding factor $(\mathrm{R})$ as the ratio of the dose rate measured after voiding $\left(D_{\text {after }}\right)$ over the dose rate measured before voiding $\left(D_{\text {before }}\right)$ :

$$
\mathrm{R}=\mathrm{D}_{\text {after }} / \mathrm{D}_{\text {before }}
$$

The percentage of dose reduction due to voiding is then given by $\left(\mathrm{D}_{\text {reduction }}\right)$ :

$$
\mathrm{D}_{\text {reduction }}(\%)=(1-\mathrm{R})^{*} 100 \%
$$

This work was approved by the hospital medical research ethics committee.

\section{Results}

The measured dose rates from the patients were reduced by $12 \% \pm$ $6 \%$ with a range of $(2 \%-30 \%)$ due to voiding. We have found a wide variation for the first void time, our average time measured before the first void was $39 \pm 8$ minutes with a range of 17-68 minutes (Table 1). The ratio of the measured dose rates before and after voiding is shown in Figure 2.

It is also worth to mention that we have excluded certain number of patient data from our study because the measured dose rate after voiding was higher than the one measured before. We concluded that these patients had accidentally contaminated their clothes with radioactive urine during voiding, which caused the dose rate after voiding to be higher.

The measured dose rate per unit activity measured from 50 patients included in this study was $93.7 \pm 14(\mu \mathrm{Sv} / \mathrm{hr} / \mathrm{GBq})$ (Table 1$)$, this value is in close agreement with the value proposed by the AAPM Task Group (92 $\mu \mathrm{Sv} / \mathrm{hr} / \mathrm{GBq})$ [1].

Figure 3 shows the measured dose rates before and after voiding from the 50 patients in this study as a function of the time corrected injected activity. 
Page 3 of 4

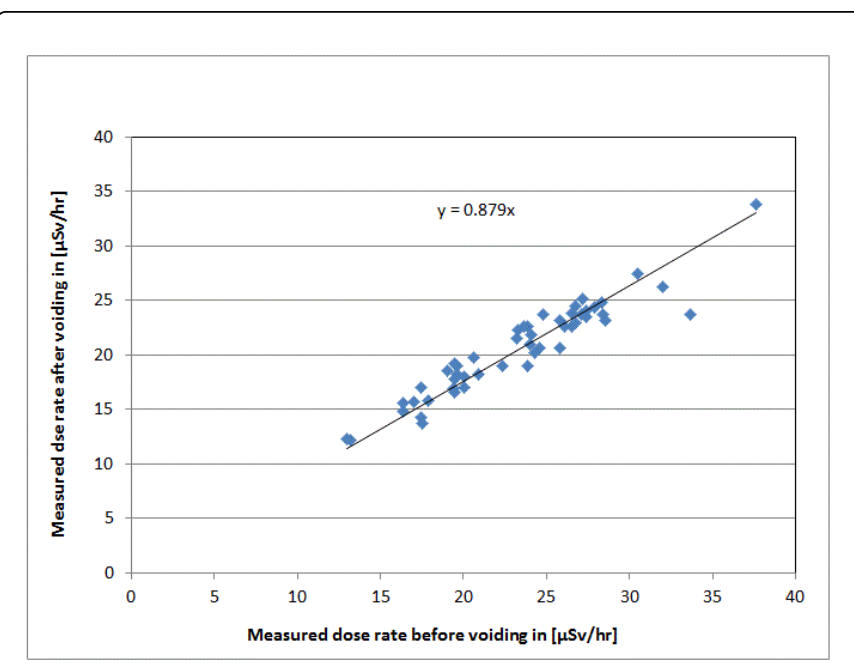

Figure 2: Measured dose rate after voiding as a function of the dose rate before voiding.

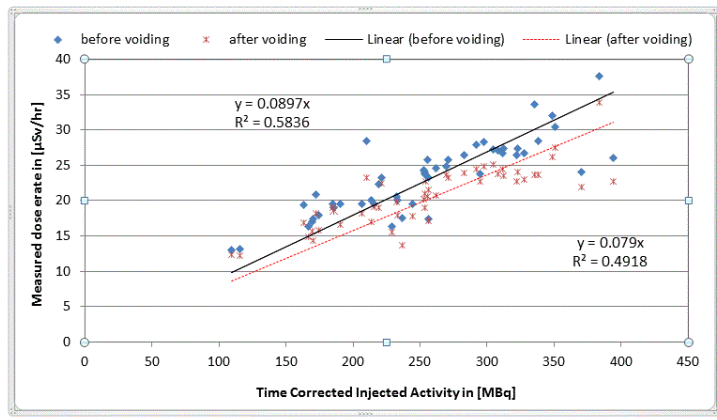

Figure 3: The measured dose rate in $[\mu \mathrm{Sv} / \mathrm{hr}]$ before and after voiding as a function of the time corrected injected ${ }^{18} \mathrm{~F}-\mathrm{FDG}$ activity in $[\mathrm{MBq}]$.

\section{Discussion}

The American association of physicist in medicine (AAPM) recommends the value of $15 \%$ of dose reduction due to bladder voiding and uses this percentage in their shielding calculations for imaging and uptake rooms design of the PET/CT suite in clinical environment [1].

Our results are in agreement with the study by Bach-Gansmo et al. they found that urinary FDG excretion to be highly variable with a range of $5.7 \%$ to $15.2 \%$ of the injected dose. They measured the urine radioactivity collected from 20 patients undergoing PET/CT imaging [2].

Hays and segall reports that bladder radiation dose will be much reduced if the patient voids early after FDG administration. They recommend that, when feasible high fluid intake and early and frequent voiding after FDG administration be encouraged [3].
The hydrated patients had a higher excretion of FDG than dehydrated patients $16.98 \%$ versus $14.27 \%$, and the volume of urine voided was significantly higher $(\mathrm{p}<0.020)[4]$.

The amount of voided activity will contribute to the dose reduction in the bladder of the patient. Jones et al. reports reduction in the order of $15 \%$ of the injected activity for the first 2 hours post injection of the FDG [5].

Mejia et al. reports a mean percentage of injected activity excreted to the bladder at 2 hours void time of $21.2 \%$ and after 1 hour to be around $13.3 \%$ [6].

The initial voiding time seems to play a role in the dose calculations to the bladder wall; the optimum initial voiding time to deliver the lowest dose according to the traditional MIRD static bladder model is 40 minutes [7].

In a study by Dowd et al. on 302 adult subjects in five-year period indicate that when the bladder is large at the time of injection, the dose to the bladder is greatly reduced. And the optimal voiding time using the dynamic bladder model is 80 minutes [8].

\section{Conclusion}

Patient bladder voiding before scanning reduced the measured dose rate at one meter from the patient by about $12 \%$ for the patients' population under study, which is an agreement with other published studies (AAPM Task Group 108).

The presented information will benefit medical physicist working in nuclear medicine, radiation safety policy makers and regulators.

\section{Acknowledgements}

The authors wish to thank Mr. Saad Alqahtani, Mrs. Huda Alnufaie and Mr.Faris Almalki, who, in part, provided that data.

\section{Conflict of Interest}

There is no conflict of interest.

\section{References}

1. Madsen MT, Anderson JA, Halama JR, Kleck J, Simpkin DJ, et al. (2006) AAPM Task Group 108: PET and PET/CT Shielding Requirements. Medical Physics 33: 4-15.

2. Bach-Gansmo T, Dybvik JA, Adamsen TC, Naum A (2012) Variation in urinary excretion of FDG, yet another uncertainty in quantitative PET. Acta Radiol Short Rep 1: 8.

3. Hays MT, Segall GM (1998) A mathematical model for the distribution of Fluorodeoxyglucose in humans. J Nucl Med 40: 1358-1366.

4. Moran JK, Lee HB, Blaufox DM (1999) Optimization of urinary FDG excretion during PET imaging. J Nucl Med 40: 1352-1357.

5. Jones SC, Alavi A, Christman D, Montanez I, Wolf AP, et al. (1982) The Radiation Dosimetry of 2-[F-18]Fluoro-2-Deoxy-D-Glucose in Man. J NucI Med. 23: 613-617.

6. Mejia AA, Nakamura T, Masatoshi I, Hatazawa J, Masaki M, et al. (1991) Estimation of absorbed doses in humans due to intravenous administration of Fluorine-18-Fluorodeoxyglucose in PET studies. J Nucl Med 32: 699-706.

7. Wu TH, Liu RS, Dong SL, Chung YW, Chou KL, et al. (2002) Dynamic evaluation of absorbed dose to the bladder wall with a ballon-bladder phantom during a study using 18-F fluorodeoxyglucose positron emission imaging. Nucl Med Commun 23: 749-755. 
Citation: Soliman K, Alenezi A (2016) Effect of patient bladder voiding on radiation dose rates measured around patients undergoing PET/CT imaging using ${ }^{18}$ F-FDG. J Nucl Med Radiat Ther 7: 272. doi:10.4172/2155-9619.1000272

Page 4 of 4

8. Dowd MT, Chen CT, Wendel MJ, Faulhaber PJ, Cooper MD (1991)

Radiation dose to the bladder wall from 2-[18F] fluoro-2-deoxy-Dglucose in adult humans. J Nucl Med 32: 707-712. 